\title{
High Contrast BSE Imaging under a Ultra Low Voltage Condition by FE-SEM with Energy Filtering
}

\author{
Y. Hashimoto ${ }^{1}$, T. Matsuzaki ${ }^{1}$, H. Ito $^{1}$, M. Konno ${ }^{1}$, and S. Takeuchi ${ }^{2}$
}

1. Global Application Center, Naka Manufacturing Division, Hitachi High-Technologies, 11-1, Ishikawa, Hitachinaka, Ibaraki, 312-0057, Japan

2. Advanced Microscope System Design Department, Naka Division, Nanotechnology Products Business Group, Hitachi High-Technologies, 882, Ichige, Hitachinaka, Ibaraki, 312-8504, Japan

BSE imaging is a useful method to obtain compositional information, and also to reduce charging effect. Demands for compositional characterization at specimen surface are increasing in the field of material science and engineering. In order to characterize compositional structure at specimen surface, Low Loss BSE (LLE) imaging is suitable because of the reduction of the information generated by multiple inelastic scattered electrons [1]. BSE imaging using Ultra Low Voltage (ULV) condition may also suitable because of the reduction of the penetration depth of incident electron beam. However, it is difficult to obtain high contrast BSE image by conventional methods. To address this challenge, we developed a method of filtering signal to the top detector of the Hitachi SU8000 FE-SEM. It is necessary to study imaging parameters to obtain high quality, high contrast BSE image under ULV condition because the signal behavior at ULV condition does not follow the conventional theory used over $1 \mathrm{kV}$ [2]. Here we report the result of fundamental experiments using a test specimen and some applications of this technology for advanced material.

To control the signal detected by the top detector, an energy filter is used. Figure 1 shows a schematic of the energy filter with the top detector in SU8000. The filter allows only electrons with higher energy than the filtering voltage to be detected. High angle BSE (HA-BSE) signal is detected by the top detector without filtering voltage, and LLE signal is detected by the top detector with an appropriate filtering voltage. When decelerating field condition is used and an appropriate filtering voltage is applied, BSE signal is detected by the top detector.

A fundamental test of the effect of energy filtering on BSE imaging using ULV was carried out using a basic test specimen. The test specimen consists of four pieces of Si wafer, and $50 \mathrm{~nm}$ thick films of three types of materials with different atomic weights $(\mathrm{C}, \mathrm{Cr}, \mathrm{Pt})$ are deposited on three pieces. Figure 2 shows the BSE images of the test specimen at $300 \mathrm{~V}$. In the conventional BSE image detected by upper detector (a), the contrast between $\mathrm{Si}, \mathrm{Cr}$, and $\mathrm{Pt}$ is not clear. However, the contrast between each material is clearly observed in top detector image with energy filtering (b). The contrast does not follow the conventional theory used over $1 \mathrm{kV}$. For example, Pt has the highest $\mathrm{Z}$ number but it has the darkest tone in the filtered image. This tendency partially conforms to a previous study [2].

Figure 3 shows the BSE images of the carbon nanotube (CNT)/ polytetrafluoroethylene (PTFE) composite film at $1 \mathrm{kV}$ (a) and at $300 \mathrm{~V}$ (b). In image (a), CNT and PTFE are identified by their topographic features but the contrast between the materials is not obvious. In image (b), the contrast between CNT and PTFE is clearly observed. By using ULV in combination with energy filtering, each material can be identified. The difference between the image quality of (a) and (b) is probably caused by the difference of the penetration depth of incident electron beam. 
Microsc. Microanal. 19 (Suppl 2), 2013 